\title{
Síndrome de shock tóxico neonatal por Streptococcus pyogenes. Reporte de caso y revisión de la literatura
}

Manuel Díaz A.

\section{Neonatal toxic shock syndrome due to Streptococcus pyogenes. Case report and literature review}

The clinical history of a newborn child who died from toxic shock syndrome (TSS) due to group A Streptococcus (GAS) was summarized. Literature was reviewed by searching different databases and other sources. Previous to our patient, there were 6 neonatal patients with TSS due to GAS published with the clinical description of the cases and three others without detailed information. In conclusion, nowadays, infection due to GAS is not a frequent devastating disease during the neonatal period, but when it is present it can cause TSS with high lethality.

Key words: Streptococcus pyogenes, meningitis, newborns, toxic shock syndrome.

Palabras claves: Streptococcus pyogenes, meningitis, recién nacidos, síndrome de shock tóxico.

\section{Introducción}

$S$ treptococcus pyogenes o $\beta$-hemolítico del grupo A de la clasificación de Lancefield, fue un problema en los comienzos del siglo pasado ${ }^{1}$, ya que era común en las infecciones puerperales y del recién nacido (RN), pero luego declinó su incidencia y, en la actualidad, es una infección poco frecuente en el período neonatal ${ }^{1}$.

La infección grave por $S$. pyogenes se ha dividido en tres formas clínicas: síndrome de shock tóxico (SST), caracterizado por hipotensión y falla multiorgánica; fascitis necrosante, en la cual hay una necrosis local extensa de los tejidos blandos subcutáneos; y la enfermedad invasora, donde $S$. pyogenes se aísla de sitios del cuerpo normalmente estériles ${ }^{2}$.

El SST es provocado por la exotoxina pirogénica de S. pyogenes, que actúa como un superantígeno, siendo capaz de estimular el sistema inmune por vía de la activación y proliferación de los linfocitos $\mathrm{T}$, e inducir una cascada de citoquinas proinflamatorias que llevan al desarrollo del shock ${ }^{3}$. Esta entidad clínica se ha reportado muy esporádicamente en neonatos ${ }^{4-10}$.

El paciente que se presenta tiene la novedad de ser el primer caso neonatal de SST por S. pyogenes que se publica en la literatura cubana e hispana en general.

\section{Caso clínico}

Un RN de 21 días de edad, de raza negra y sexo masculino, consultó de urgencia por fiebre hasta $38,5^{\circ} \mathrm{C}$, rechazo al pecho y manifestaciones catarrales respiratorias. Los antecedentes del embarazo y el parto -ocurrido a las 39 semanas de gestación- eran normales; el puntaje de Apgar fue 9-9 y pesó al nacer $2.880 \mathrm{~g}$. Egresó del hospital con lactancia materna exclusiva.

Al examen físico de reingreso se constató buena vitalidad y coloración, pesaba $3.580 \mathrm{~g}$, tenía escasa rinorrea serosa y sin otros hallazgos significativos. En la evaluación para pesquisa de sepsis se estableció: leucocitos de $8.500 / \mathrm{mm}^{3}$ (PMN 68\%), VHS $26 \mathrm{~mm} / \mathrm{h}$, examen de orina normal, examen citoquímico del LCR con 6 leucocitos $/ \mathrm{mm}^{3}, 86$ hematíes $/ \mathrm{mm}^{3}$, proteína 60 $\mathrm{mg} / \mathrm{dl}$ y glucosa $26 \mathrm{mg} / \mathrm{dl}$; radiografía de tórax $\sin$ alteraciones pleuro-pulmonares. Por sus buenas condiciones clínicas y exámenes de laboratorio dentro de rangos normales, se ingresó sin tratamiento antimicrobiano.

Al segundo día reapareció la fiebre, hasta $38,0{ }^{\circ} \mathrm{C}$ y se informó un resultado positivo en el urocultivo, (más tarde, se identificó una Serratia sp), por lo que se inició tratamiento con gentamicina ( $7 \mathrm{mg} / \mathrm{kg} / \mathrm{d}$ áa $)$ ev. El cultivo del LCR fue negativo. Entre el tercer y cuarto día apareció deterioro de su condición clínica, con quejido, rechazo de la lactancia materna, adinamia e hiporreflexia, somnolencia con irritabilidad, distensión abdominal y vómitos, signos de dificultad respiratoria, palidez, cianosis distal, taquicardia, oligoanuria y signos de hipoperfusión tisular, con un aspecto tóxicoinfeccioso, presentando convulsiones y bregma normotenso. Reevaluado con exámenes de laboratorio, se encontró: leucocitos $12.300 / \mathrm{mm}^{3}$ (PMN 34\% con pre-
Hospital Pediátrico Universitario Juan M. Márquez Ciudad de la Habana. Cuba Servicio de Neonatología.

Recibido: 29 de abril de 2007 Aceptado: 1 de octubre de 2007

Correspondencia a: Manuel Díaz Álvarez mfdiaz@infomed.sld.cu 


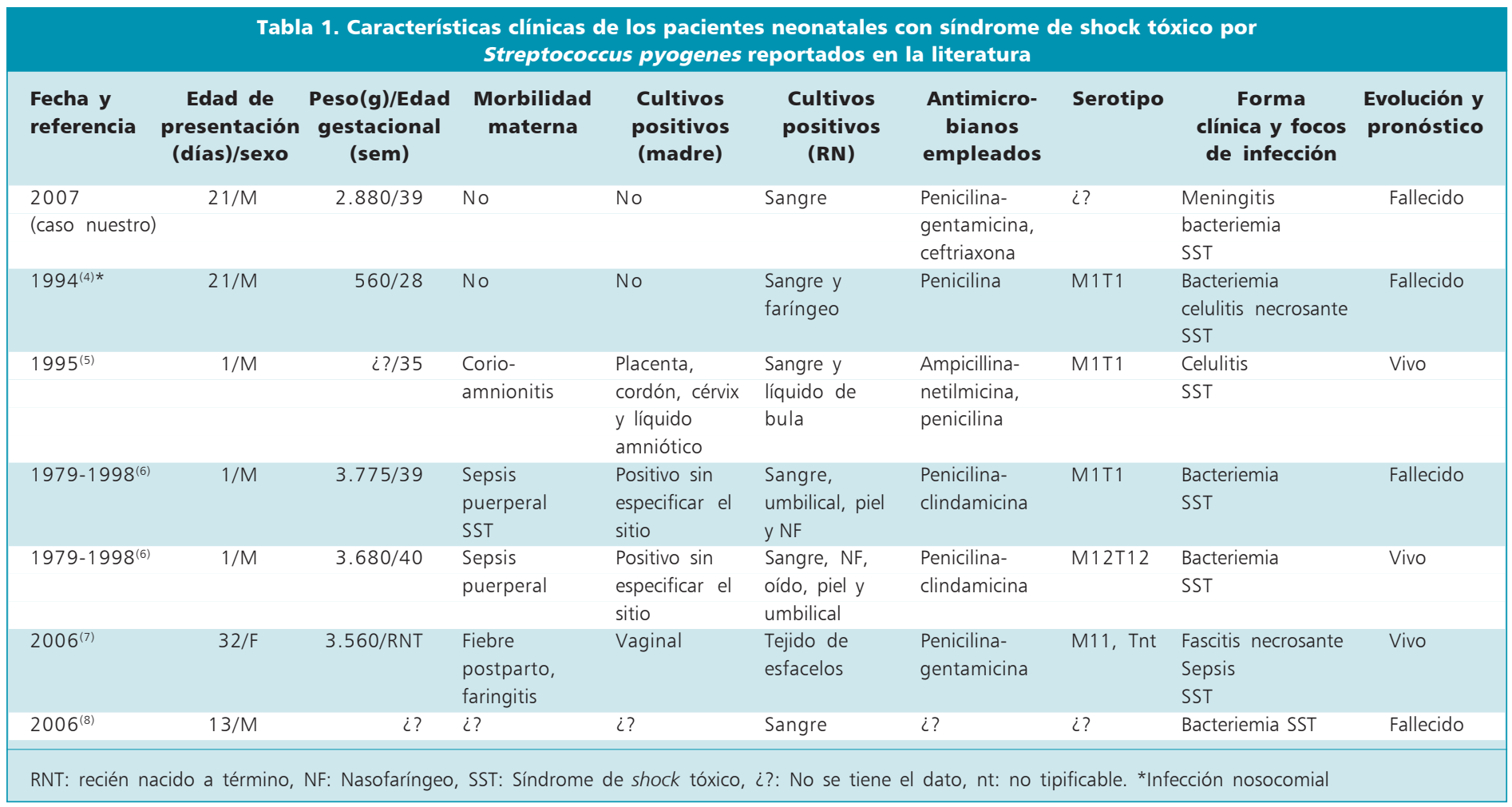

sencia de granulaciones tóxicas), VHS $16 \mathrm{~mm} / \mathrm{h}$, plaquetas de $110.000 / \mathrm{mm}^{3}$, gasometría con acidosis metabólica; examen de orina normal, en la radiografía de tórax aparecieron imágenes compatibles con síndrome de dificultad respiratoria del adulto. El paciente fue apoyado con cuidados intensivos: reposición de volumen, inotrópicos, ventilación mecánica, inmunomoduladores (inmunoglobulina IV y corticosteroides) y recibió fenobarbital. Se indicó penicilina G sódica (200.000 U/kg/día) ev al conocerse resultado del hemocultivo de ingreso positivo a $S$. pyogenes. A las 48 horas de iniciado el tratamiento antimicrobiano se repitió la punción lumbar y se informó el examen citoquímico del LCR con 47 leucocitos $/ \mathrm{mm}^{3}, 2.304$ hematíes $/ \mathrm{mm}^{3}$, proteína $87 \mathrm{mg} / \mathrm{dl}$ y glucosa $18 \mathrm{mg} / \mathrm{dl}$. El cultivo del LCR de la segunda punción lumbar resultó negativo.

En los días posteriores, el paciente presentó un deterioro clínico progresivo con aparición de convulsiones, por lo que se incrementó la dosis de anticonvulsivantes, cayó en coma profundo, con midriasis y respuesta pupilar débil, postura de descerebración y bregma tenso. En el ultrasonido cerebral se apreciaba una hemorragia periventricular e intraventricular. Se inició tratamiento para la hipertensión intracraneal y se cambió los antimicrobianos iniciales por ceftriaxona
(130 mg/kg/día) ev. Sin embargo, el RN falleció. En la necropsia se comprobaron signos de meningitis con hemorragias y daño cerebral grave, signos de shock séptico y pulmones compatibles con el síndrome de dificultad respiratoria del adulto.

\section{Discusión}

El paciente que relatamos es el primer caso neonatal reportado en la literatura cubana e hispana que reúne los criterios clínicos de un SST por S. pyogenes en neonatos, de acuerdo con la definición establecida por el Comité en Enfermedades Infecciosas para las Infecciones Invasoras Graves por $S$. pyogenes ${ }^{11}$; esto es, aislado de $S$. pyogenes a partir de un sitio normalmente estéril (usualmente sangre o LCR); un cuadro clínico de shock, con necesidad de aportes de volumen y de inotrópicos; más falla multiorgánica, con al menos dos problemas presentes que involucraron clínicamente un SDRA en el RN y la afectación renal con oligoanuria, todo lo cual se comprueba en la necropsia, donde se verificó una necrosis cerebral, junto al compromiso pulmonar y hepato-renal como lo más significativo.

El presente caso es relevante por tratarse de un SST en el curso de una infección por $S$. pyogenes en 
neonatos, un evento muy poco frecuente, aunque se han publicado algunos casos en la literatura internacional. En la revisión de Miyairi y cols ${ }^{12}$, de infección invasora por S. pyogenes, que abarcó la literatura médica en lengua inglesa desde 1966, se presentan 39 RNs con esta infección, algunos de los cuales cursaron con SST, pero no se puede definir cuáles fueron los casos específicos que presentaron este cuadro. Además de esto, la definición operacional de este síndrome se estableció hace pocos años, después de la primera descripción por Cone y cols, en $1987^{13}$, por lo que algunos pacientes publicados anteriormente pudieron no haber sido reconocidos como SST.

Al revisar la literatura científica acerca de las infecciones invasoras causadas por S. pyogenes, pudimos identificar seis RN con información detallada acerca de sus características clínicas de presentación y evolución, y que tuvieron SST en el curso de una infección invasora por $S$. pyogenes; ninguno de ellos presentó meningitis (Tabla 1). En un paciente con dos semanas de edad reportado por Belani ${ }^{14}$, con bacteriemia y celulitis, se recuperó una cepa de $S$. pyogenes productora de exotoxina $\mathrm{A}$ y $\mathrm{B}$, las que son reconocidas como potentes estimuladoras e inductoras de shock, pero la información aportada del paciente no refleja que presentara los criterios de SST. En otros artículos publicados, en los que no se ofrece información en particular de los casos, se pudo identificar un RN fallecido que cursó con meningitis y SST por $S$. pyogenes ${ }^{9}$, más dos neonatos con SST (uno fallecido al nacer y otro egresado vivo), en los que se señala que experimentaran una infección invasora por $S$. pyogenes, sin precisar qué tipo de infección presentaban ${ }^{10}$.

Definitivamente, son pocos los RNs afectados de infección invasora por $S$. pyogenes y SST, reportados en la literatura pues, contabilizando nuestro caso, suman 10 pacientes, de los cuales sólo dos -incluido el nuestro- presentaron SST en el curso de una sepsis con un foco secundario meníngeo. Nuestro paciente tiene la particularidad de que, en un inicio tuvo un LCR negativo, pero, posteriormente, a partir de una bacteriemia por $S$. pyogenes detectada en el hemocultivo de ingreso, alcanzó al SNC, desencadenándose, coincidentemente, el SST.

Es notable que en cuatro pacientes, de los cinco de quienes se ofrece información, las cepas aisladas eran de los serotipos M1 y M12, las cuales se han vinculado a la ocurrencia de $\mathrm{SST}^{3}$.

El SST se presenta en el curso de una infección invasora por $S$. pyogenes, en sus diversas formas clínicas, tanto sistémicas como focales ${ }^{10,15-16}$. En el RN puede manifestarse como una infección de inicio temprano ( $\leq 4$ días), o tardía ( $>4$ días). El mecanismo de adquisición de la infección de inicio temprano es por transmisión vertical, relacionada a eventos perinatales tales como sepsis puerperal y corioamnionitis, mientras que la infección de inicio tardío se transmite en forma horizontal, siendo, habitualmente, puerta de entrada la piel y las mucosas, que han sido vulneradas facilitando la aparición de una infección focal. Estas características se ven reflejadas en los pacientes descritos en la Tabla 1.

Penicilina sigue siendo el antimicrobiano de elección para tratar los pacientes, aún en las infecciones graves, pues no se reporta hasta la actualidad resistencia in vitro a ella. Se recomienda adicionar clindamicina a penicilina en los estadios iniciales de la infección grave por $S$. pyogenes, pues esta lincosamida tiene, en teoría, la capacidad de reducir la producción de toxina estreptocóccica, potenciar la fagocitosis y alcanzar elevada concentración tisular ${ }^{17}$. La inmunoglobulina intravenosa se ha utilizado como terapia inmunomoduladora del shock con resultados favorables $^{5,18}$. Aun así, la evolución fatal en un paciente con SST por $S$. pyogenes es un evento muy probable, dada la gravedad de esta condición clínica. En los 10 pacientes que se presentan, seis fallecieron. La presencia de SST en la infección invasora por $S$. pyogenes es un factor de riesgo y se asocia, significativamente, con alta letalidad ${ }^{9,19-20}$.

Concluimos que, al considerar todas las publicaciones citadas, aunque en la actualidad las infecciones invasoras causadas por $S$. pyogenes no son ya un problema frecuente en el período neonatal, si se presentan, pueden cursar con SST cuyo pronóstico es habitualmente fatal.

\section{Resumen}

Se realizó un resumen de la historia clínica de un recién nacido $(\mathrm{RN})$ fallecido por síndrome de shock tóxico (SST) a Streptococcus pyogenes y se revisó la literatura existente al respecto mediante la búsqueda en distintas bases de datos y otras fuentes. Previo a nuestro paciente se han publicado seis RNs con SST por $S$. pyogenes con la descripción clínica de los mismos y otros tres RNs sin información detallada de los casos. Se concluye que, en la actualidad, la infección por $S$. pyogenes no es ya un flagelo en el período neonatal, pero cuando se presenta, puede provocar SST con elevada letalidad. 


\section{Referencias}

1.- Bizzarro M J, Raskind C, Baltimore R S, Gallagher P G. Seventy-five years of neonatal sepsis at Yale: 1928-2003. Pediatrics 2005; 116: 595-602.

2.- Chuang Y Y, Huang Y C, Lin T Y. Toxic shock syndrome in children: epidemiology, pathogenesis, and management. Pediatr Drugs 2005; 7: 11-25.

3.- Stevens D L. Invasive streptococcal infections. J Infect Chemother 2001; 7: $69-80$

4.- Campbell J R, Arango C A, García-Prats J A, Baker C J. An outbreak of M serotype 1 group A Streptococcus in a neonatal intensive care unit. J Pediatr 1996; 129: 396-402.

5.- Mahieu L M, Holm S E, Goossens H J, Van Acker K J. Congenital streptococcal toxic shock syndrome with absence of antibodies against streptococcal pyrogenic exotoxins. J Pediatr 1995; 127: 987-9.

6.- Verboon-Maciolek M A, Krediet T G, van Ertbruggen I, Gerards L J, Fleer A. Severe neonatal group A streptococcal disease. Eur J Pediatr 2000; 159: 450-2.

7.- Davey C, Moore A M. Necrotizing fasciitis of the scalp in a newborn. Obstet Gynecol 2006; 107 (2 Pt 2): 461-3.

8.- Kadivar M, Haghi S, Mahjoub F. A neonate with septic shock and multiorgan dysfunction because of group A streptococci and review of the literature Iran. J Pediatr 2006; 16 (4): 501-6.

9.- Eriksson B K G, Andersson J, Holm S E, Norgren M. Epidemiological and clinical aspects of invasive group A streptococcal infections and the streptococcal toxic shock syndrome. Clin Infect Dis 1998; 27: 1428-36.

10.- Svensson N, Oberg S, Henriques B, Holm S, Kallenius G, Romanus V, et al. Invasive group A streptococcal infections in Sweden in 1994 and 1995: epidemiology and clinical spectrum. Scand J Infect Dis 2000; 32: 609-14.

11.- Committee on Infectious Diseases of the American Academy of Pediatric. Severe invasive group A streptococcal infections: A subjet review. Pediatrics 1998; 101: 136-40.

12.- Miyairi I, Berlingieri D, Protic J, Belko J. Neonatal invasive group A streptococcal disease: case report and review of the literature. Pediatr Infect Dis J 2004; 23: 161-5.

13.- Cone A L, Woodard D R, Schlievert P M, Tomory G S. Clinical and bacteriological observations of a toxic shock-like syndrome due to Streptococcus pyogenes. N Engl J Med 1987; 317: 146-9.

14.- Belani K, Schlievert P M, Kaplan E L, Ferrieri P. Association of exotoxinproducing group A streptococci and severe disease in children. Pediatr Infect Dis J
1991; 10: 351-4.

15.- Huang Y C, Hsueh PR, Lin T Y, Yan D C, Hsia S H. A family cluster of streptococcal toxic shock syndrome in children: Clinical implication and epidemiological investigation. Pediatrics 2001; 107: 1181-4.

16.- Chiang M C, Jaing T H, Wu C T, Hsia S H, Chiu C H. Streptococcal toxic shock syndrome in children without skin and soft tissue infection: Report of four cases. Acta Paediatr 2005; 94: 763-5.

17.- Steer A C, Danchin M H, Carapetis J R. Group A streptococcal infections in children. J Paediatr Child Health 2007; 43: 203-13.

18.- Kaul R, McGeer A, Norrby-Teglund A, Kotb M, Schwartz B, O'Rourke K, et al. Intravenous immunoglobulin therapy for streptococcal toxic shock syndrome - a comparative observational study. The Canadian Streptococcal Study Group. Clin Infect Dis 1999; 28: 800-7.

19.- Davies H D, McGeer A, Schwartz B, Green K, Cann D, Simor A E, et al. Invasive group A streptococcal infections in Ontario, Canada. N Engl J Med 1996; 335: 547-54.

20.- Vallalta Morales M, Soriano Navarro C J, Salavert Lletí M, Montero Alonso M, Pérez Bellés C, López Aldeguer J, et al. Group A streptococcal bacteremia: outcome and prognostic factors. Rev Esp Quimioterap 2006; 19: 367-75. 